\title{
Laterality effects in the recognition of depth-rotated novel objects
}

\author{
KIM M. CURBY \\ Vanderbilt University, Nashville, Tennessee \\ WILLIAM G. HAYWARD \\ Chinese University of Hong Kong, Hong Kong \\ and \\ ISABEL GAUTHIER \\ Vanderbilt University, Nashville, Tennessee
}

\begin{abstract}
The dissociable neural subsystems theory proposes that left-hemisphere (LH) performance is dominated by a viewpoint-invariant (VI) recognition subsystem, whereas right-hemisphere (RH) performance is dominated by a viewpoint-dependent (VD) subsystem (Marsolek, 1999). Studies supporting this theory have used familiar objects and, therefore, may have been confounded by characteristicsbeyond perceptual features. Experiment 1, a lateralized sequential-matching task with novel objects, showed VD recognition in both hemispheres. In Experiment 2, some participants learned semantic associations for four novel objects, whereas others were exposed to the novel objects without the semantic associations. Both groups later performed a depth-rotated lateralized sequential-matching task. The participants who had learned semantic associations showed greater VD performance in the RH than in the LH; however, the participants in the control group showed equivalent VD performance in both hemispheres. The results suggest that hemispheric differences in VD performance may be partially attributable to an LH advantage for semantic processing.
\end{abstract}

How much do object recognition abilities depend on the specific view of an object being observed? This question has been the topic of a lively theoretical debate in which there have been two opposing schools of thought. Viewpoint-invariant (VI) theories of object recognition, such as the recognition-by-components theory (Biederman, 1987), suggest that recognition processes are fairly robust to changes in the viewpoint of an object. One way this can be achieved is through the use of structural descriptions composed of VI three-dimensional (3-D) parts and the relationships between them (Biederman \& Cooper, 1991b, 1992; Cooper, Biederman, \& Hummel, 1992). Therefore, such a theory predicts that, providing the same structural description can be retrieved, recognition will be VI. In contrast, viewpoint-dependent (VD) theories propose that object representations are collections of views that depict the appearance of an object from specific viewpoints (e.g., Poggio \& Edelman, 1990; Riesenhuber \& Poggio, 1999) and, therefore, predict that chang-

This study was supported by Grant CUHK4332/00H from the Research Grants Council of the Hong Kong SAR to W.G.H. and by NSF Grant 0091752 to I.G. We thank Chad Marsolek, Michael Tarr, Steven Most, and one anonymous reviewer for helpful comments on earlier versions of this manuscript. We also thank Danielle Brown for help with data collection. Correspondence concerning this article should be addressed to K. M. Curby, Department of Psychology, 301 Wilson Hall, Vanderbilt University, Nashville, TN 37203 (e-mail: kim.curby@ vanderbilt.edu). ing the viewpoint of an object to one that is less familiar will impair recognition performance. One reason the debate has been difficult to resolve is that both patterns of performance have been demonstrated in different situations (Biederman \& Bar, 1999; Biederman \& Gerhardstein, 1993; Hayward \& Tarr, 1997; Tarr, 1995; Tarr \& Gauthier, 1998; Tarr \& Pinker, 1989, 1990; Tarr, Williams, Hayward, \& Gauthier, 1998).

Recently, it has been suggested that recognition may be neither inherently VI nor VD but that, depending on the specifics of the task, recognition may lie on a continuum of viewpoint specificity (Farah, 1992; Hayward \& Williams, 2000; Tarr \& Bülthoff, 1995). Marsolek (1999) has proposed that distinct recognition processes are used, depending on the requirements of a task. According to the dissociable neural subsystems (DNS) theory, the recognition of abstract categories is performed by an abstract visual form subsystem, which uses a parts-based strategy to process relatively invariant input information. This subsystem is believed to function more effectively in the left hemisphere (LH). In contrast, a specific visual form subsystem would underlie recognition of specific instances. This subsystem would use a holistic strategy to process visual inputs and is thought to function more effectively in the right hemisphere (RH).

DNS theory has been applied to a variety of domains, including the recognition of words (Marsolek, Kosslyn, \& Squire, 1992; Marsolek, Squire, Kosslyn, \& Lulenski, 
1994), letter-like shapes (Marsolek, 1995), and pseudowords (Burgund \& Marsolek, 1997); most recently, it has been applied to object recognition (Burgund \& Marsolek, 2000; Marsolek, 1999). A series of divided visual field repetition-priming experiments was performed with familiar objects: Objects were primed by the same exemplar, a different exemplar with the same name, or the word associated with the name of the test object (Marsolek, 1999). Priming was greater following same-exemplar primes than following different-exemplar primes in the $\mathrm{RH}$, whereas no difference was observed when stimuli were presented to the LH. These results suggest that priming was exemplar abstract (yet visual) when test objects were presented to the LH but exemplar specific when test objects were presented to the RH.

Further experiments suggested that this model can account for differences between VI and VD patterns of performance (Burgund \& Marsolek, 2000). A lateralized priming task was used in which the prime and the test views contained either the same or a different set of parts. In a study phase, the participants listened to the name of the object; they then viewed the object and, finally, rated the object in terms of how much they liked it. In the subsequent test phase, the participants were required to name the object, briefly presented to the left or right of fixation. The investigators suggested that the holistic-processing style believed to be used by the $\mathrm{RH}$-dominant specific subsystem should result in a recognition cost when the test object was rotated relative to the prime, regardless of whether the same parts were visible, since the rotation would result in a change to the whole-based information. However, the proposed partsbased-processing style of the LH-dominant abstract subsystem should result in a lesser cost for rotations in which the parts-based visual information remained unchanged. In line with the core predictions of DNS theory (Marsolek, 1999), the results showed that priming mediated by the RH was VD, whereas LH priming was VI. However, contrary to other predictions made by this theory, the relative visibility of the parts in the presented views failed to change the nature of these results in either hemisphere.

The results of Marsolek's (1999; Burgund \& Marsolek, 2000) studies suggest that the VI/VD debate in object recognition research may be due to competing hemispherically-lateralized systems. However, there are two difficulties in relating Marsolek's priming studies to those at the heart of the object recognition debate. First, object recognition theorists (e.g., Biederman, 1987; Hummel \& Stankiewicz, 1998) typically predict that VI performance, if found, will hold only when the same parts and spatial configuration are observed across views. As was reported earlier, Burgund and Marsolek (2000) investigated this issue and found similar levels of priming in both the $\mathrm{LH}$ and the $\mathrm{RH}$ for rotations that did and did not show the same parts across both views. To explain their results, they argued that the features encoded by the abstract subsystem are more general than the parts.
No current theory of object recognition proposes components that would be compatible with this conjecture.

Second, although the DNS theory makes clear predictions for recognition of familiar objects, predictions for novel objects are not so clear. Many object recognition experiments use novel objects, in part because the multipleviews and the structural description models make similar predictions for familiar objects experienced from a number of viewpoints (Hayward \& Tarr, 1997; Srinivas, 1995; Tarr, 1995). However, Burgund and Marsolek (2000) have argued that novelty may be critical in determining the relative contributions of the abstract and the specific subsystems. They noted that novel information is typically found in the holistic structures of objects because even novel objects are composed of familiar features at some level (e.g., volumes). Earlier work had suggested that the specific subsystem stores novel information more effectively than does the abstract subsystem (Marsolek, Schacter, \& Nicholas, 1996), suggesting that the specific system has an advantage and contributes more to the recognition of novel objects. Burgund and Marsolek (2000) argued that this predisposition for the specific subsystem to process novel information more effectively may explain why so many experiments employing novel objects have found VD patterns of performance. However, this issue has not been specifically tested in the context of the DNS theory.

\section{EXPERIMENT 1}

The aim of Experiment 1 was to examine systematically the nature of lateralized priming on recognition of novel objects and, in doing so, to evaluate the DNS theory as at least a partial explanation for the viewpoint debate in object recognition.

We reasoned that the use of novel objects should provide better insight into these visual subsystems because there would be less of a confound from characteristics beyond perceptual features (e.g., semantic associations). We also manipulated the parts that were shared between different views of each object. As was noted, the use of novel objects means that a variety of predictions are possible. On the basis of previous findings (Burgund \& Marsolek, 2000), we can predict that if novelty does not affect the operation of the LH and RH subsystems, object rotations should disadvantage the RH-dominant specific subsystem, but not the LH-dominant abstract subsystem, and that this RH disadvantage should occur regardless of whether the rotations result in a change to the visible parts. However, the use of novel objects that are relatively free from semantic and conceptual information may allow for the demonstration of a pattern of performance more consistent with the proposed processing nature of these "visual" subsystems. That is, the partsbased processing nature of the LH-dominant subsystem results in its experiencing a reduced cost when objects are rotated in such a way that there is no change to the visible parts. This pattern of performance would also be 
more consistent with previous studies reporting VI performance (Biederman, 1987; Hummel \& Stankiewicz, 1998). Alternatively, the use of novel objects may allow the specific subsystem to dominate the recognition decision, as has been proposed by Burgund and Marsolek (2000), with both hemispheres experiencing the same cost, due to the change in whole-based information.

We used 10 novel objects, each composed of five distinct volumetric shapes, as targets in a divided visual field paradigm. The participants performed same-different judgments based on a procedure used by Biederman and Gerhardstein (1993). In the no-rotation condition, the object was shown in exactly the same viewpoint on each presentation. In the no-change rotation, the object was rotated $45^{\circ}$, and no parts were occluded (nor were any previously occluded parts made visible). In the change rotation, the object underwent a $45^{\circ}$ rotation that occluded previously visible parts or showed previously occluded parts.

\section{Method}

Participants. Fifty strongly right-handed (laterality quotient greater than 92, Edinburgh Handedness Inventory; Oldfield, 1971) undergraduate students (14 males) at the University of Wollongong, Australia, participated in order to fulfill a course requirement. All reported normal or corrected-to-normal vision. The participants gave informed consent according to procedures approved by the University of Wollongong Ethics Committee.

Materials. Ten computer-generated 3-D target objects were created on a Macintosh computer, using 3-D modeling software (Strata Vision 3-D). The objects are shown in Figure 1. Each object consisted of a large central purple volume and four small adjoining lime green parts.

For each object, a number of target views were created. Four rotation pairs were created for each object, with members of each pair separated by a $45^{\circ}$ rotation (see Figures $1 \mathrm{~B}$ and $1 \mathrm{C}$ for examples).
Two of the pairs were used in the change-rotation condition: one in which the rotation resulted in the inclusion of one or two previously occluded parts, and one in which the rotation resulted in the occlusion of one or two previously visible parts. The other two pairs were used in the no-change-rotatio $\mathrm{n}$ condition: One pair of images showed the object with the same two parts, and the other pair showed the object with the same three parts.

Photo-realistic 8-bit renderings of these objects were created using realistic lighting but no cast shadows, and each image was saved as both a $70 \times 70$ and a $140 \times 140$ pixel size image. The experiment was run on an Apple Macintosh with a 15-in. color monitor at a resolution of $640 \times 480$ pixels.

Design and Procedure. Each trial began with a fixation cross for $2,000 \mathrm{msec}$, followed by the first object for $750 \mathrm{msec}$, a fixation cross for $500 \mathrm{msec}$, a letter for $10 \mathrm{msec}$, and on $83 \%$ of the trials, a second object presented to the right or the left of the center of the screen for $150 \mathrm{msec}$. The degree of visual angle subtended horizontally and vertically by the first object, the letter, and the second object was $2^{\circ}, 0.5^{\circ}$, and $4^{\circ}$, respectively. The distance from the fixation point to the center of the second object was $4^{\circ}$, with an inner edge never closer than $2^{\circ}$. Response latencies were recorded from the onset of the second object. The second object was presented briefly $(150 \mathrm{msec})$, to prevent fixation on the stimulus (Carpenter, 1981). To ensure central fixation, if a second object did not appear, the participants were required to report the identity of the letter. The data from the participants were included only if they satisfactorily reported the letter, when required, with an accuracy of at least $80 \%$.

The participants used a chinrest $71.5 \mathrm{~cm}$ from the screen, yielding a display area subtending approximately $2^{\circ}$ of visual angle, within which the first object appeared. The participants were told that the objects might be presented in different viewpoints, although recognition decisions were to be made solely on the basis of object identity. The participants pressed a key indicating whether the two objects were the same or different. If there was no second object, the participants typed in the letter that was presented after the first object.

Each participant performed a series of 14 practice trials. In the experiment, each of the 10 objects appeared in 10 same trials, and in 10 different trials, and in 4 trials in which no second object ap-

A)
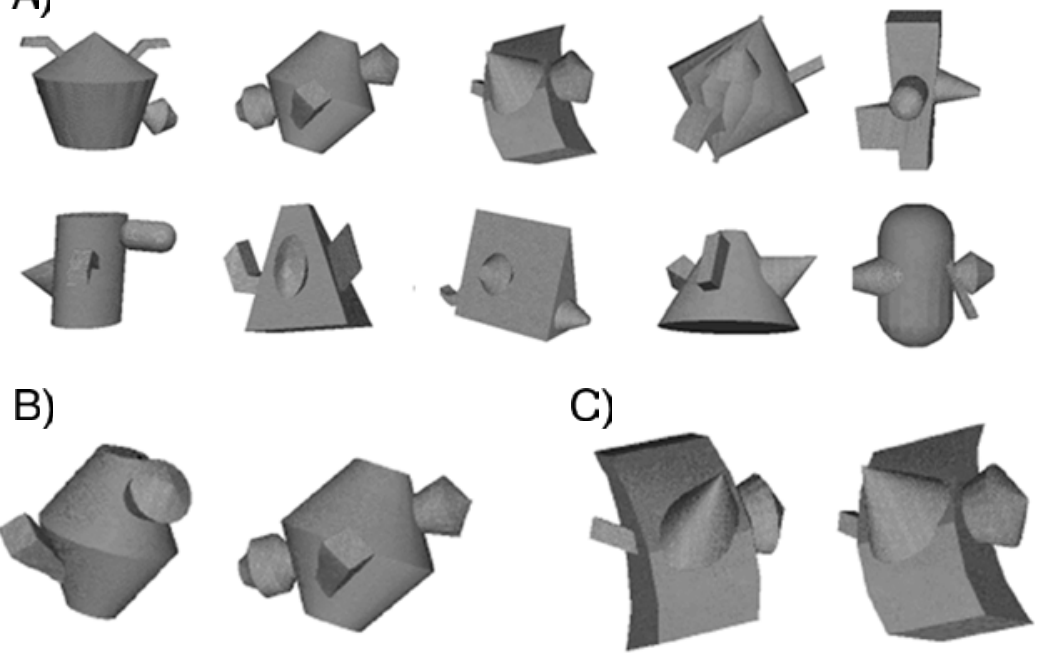

Figure 1. (A) The 10 novel objects used as stimuli in Experiment 1. Different viewpoints of the objects were used to satisfy the rotation conditions. Also shown are examples of the (B) change-rotation (inclusion) and (C) no-change-rotation, used in the same trials; $45^{\circ}$ separated each pair. 
peared, for a total of 240 trials. The same and the different trials were equally divided into two further groups, depending on which side of the screen (right or left) the second object was presented on.

Same trials were generated by pairing different viewpoints of the same object in order to satisfy one of the three rotation conditions (no-rotation, no-change-rotation, or change-rotation; Figures 1B and $1 \mathrm{C}$ ). Due to the fact that there were two types of same-parts and different-parts rotation conditions, there were twice as many trials in these conditions as in the no-rotation condition.

Different trials were generated by pairing each of the 10 viewpoints per object with one of a hundred distractor objects made using the same geons as those in the original set. Different trials were matched with same trials for the number of parts in the first and the second objects. Trial order was randomly determined, and the participants received feedback for an incorrect response after every trial.

Two different sets of distractors were used in the experiment. The first set was created using the same central volumes as those in the experimental set, with 10 new objects created for each of the existing 10 central volumes. A second set of distractor objects was created in order to make a different trial that was matched to each existing same trial (in terms of the central volume, orientation, number of parts, and viewpoint). Half the participants (25) performed trials with one of the distractor sets, whereas the other half performed trials with the other set. As will be reported in the Results section, there were statistically no differences between the different distractor sets.

\section{Results}

Trials with a reaction time (RT) greater than $4,000 \mathrm{msec}$ or less than $200 \mathrm{msec}$ were excluded from the analysis (fewer than $0.5 \%$ of the trials). Our first aim was to establish whether there were any statistical differences between the two distractor sets. The average false alarm rate $(37.3 \%$ and $37.6 \%$ for Sets 1 and 2 , respectively) and the overall sensitivity ( $1.071[S E=0.055]$ and 1.11 $[S E=0.054]$, respectively) were very similar. Sensitivity measures $\left(d^{\prime}\right)$ were calculated, and a $2 \times 3 \times 2$ mixed analysis of variance (ANOVA) was conducted on these data in order to examine differences between trials performed in the context of matched or unmatched distrac- tors. It showed no significant main effect of distractor type $(F<1)$ and no interaction with hemisphere $(F<1)$ or condition $(F<1)$. In addition, the three-way interaction between hemisphere, condition, and distractor type failed to reach significance $[F(2,48)=1.6238, p>$ .05]. The same analysis was performed on the RT data and showed similar results, with no significant main effect of distractor type $(F<1)$ and no interaction with hemisphere $(F<1)$ or condition $[F(2,48)=1.405, p>$ $.05]$. The three-way interaction also failed to reach significance $(F<1)$. Therefore, all further analyses were performed on the data collapsed over the two distractor conditions.

Mean sensitivity and RTs for trials in which the same object was shown at each presentation for the three rotation conditions are shown in Figure 2. The results were similar to those in previous studies (e.g., Biederman \& Gerhardstein, 1993; Hayward, 1998; Hayward \& Tarr, 1997). Objects were recognized most quickly and most accurately in the no-rotation condition. Objects were recognized most slowly and least accurately in the changerotation condition. Performance in the no-change-rotation condition fell between these extremes. There was an effect of viewpoint regardless of whether or not the parts changed, and this pattern was observed in both hemispheres. The effect of the no-change rotation, relative to the no-rotation, condition varied across the hemispheres, with the LH incurring a greater cost to sensitivity $\left(\Delta d^{\prime}=\right.$ $0.42)$ than did the RH $\left(\Delta d^{\prime}=0.15\right)$.

Sensitivity. The above conclusions were supported by a $2 \times 3$ (hemisphere $\times$ rotation condition) ANOVA performed on the sensitivity measure $\left(d^{\prime}\right)$, which yielded an effect of condition $\left[F(2,49)=201.84, M S_{\mathrm{e}}=33.69, p<\right.$ $.0001]$ and a significant interaction between hemisphere and condition $\left[F(1,49)=5.51, M S_{\mathrm{e}}=0.54, p<.01\right]$. There was no significant effect of hemisphere $(F<1)$.
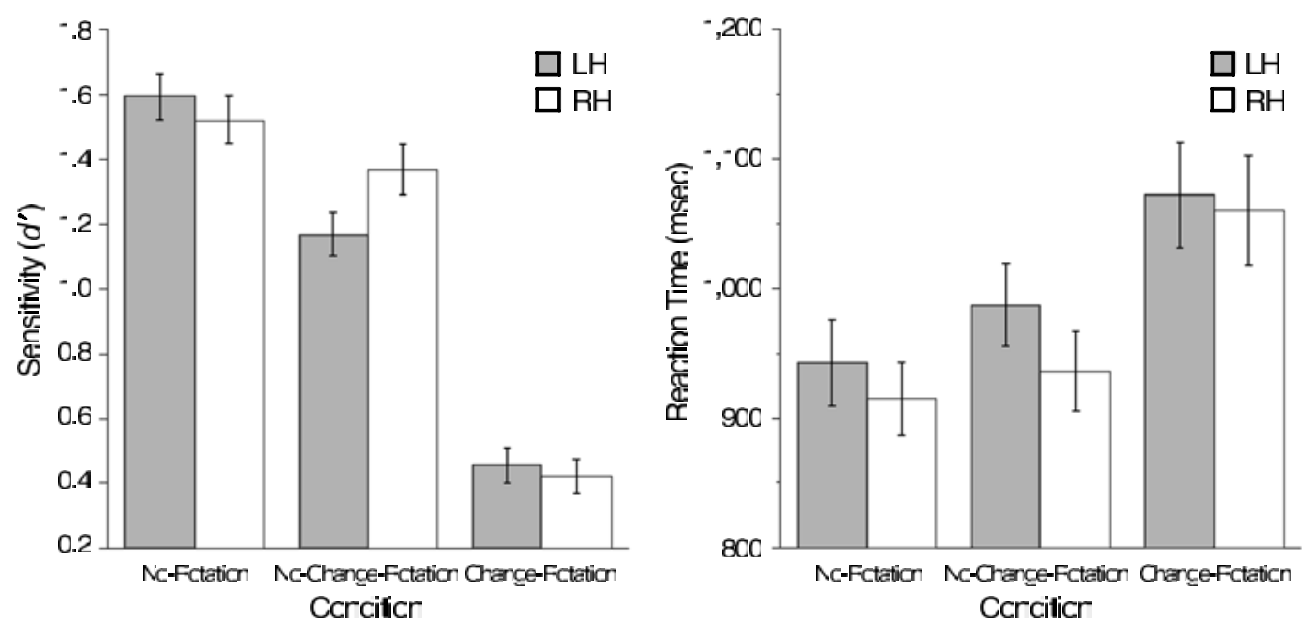

Figure 2. (A) Mean sensitivity and (B) reaction times for the recognition of novel objects presented to the left $(\mathrm{LH})$ or right hemisphere $(\mathrm{RH})$ in a sequential-matching task. Objects were presented in three rotation conditions: no rotation, rotation resulting in no change in the parts visible, and rotation resulting in a change in the visible parts. 
The interaction between hemisphere and condition appears to be due to the additional cost incurred by the $\mathrm{LH}$ in the no-change-rotation condition (see Figure 2). Planned $t$ tests performed on the difference in sensitivity between the no-change and the no-rotation conditions found that the $\mathrm{LH}[t(49)=7.142, p<.0001]$, but not the RH $[t(49)=$ $1.906, p>.05]$, incurred a cost significantly greater than zero to sensitivity in the no-change-rotation condition, as compared with the no-rotation condition. In addition, Scheffé tests showed that the change-rotation condition had significantly lower sensitivity than did the no-rotation condition in both hemispheres $(p \mathrm{~s} \leq .0001){ }^{1}$

Response time. Although more variable than sensitivity, RT followed the same pattern, with the participants performing fastest in the no-rotation condition, followed by the no-change-rotation and, finally, the change-rotation condition. RT also showed the same laterality trend as sensitivity, with the LH appearing to experience a greater cost from no-change-rotation, as compared with the norotation condition. Overall, the LH was slightly slower on average than the $\mathrm{RH}$, although there appeared to be a small speed-sensitivity tradeoff for the no-rotation and the change-rotation conditions.

A $2 \times 3$ ANOVA revealed significant effects of condition $\left[F(3,49)=30.871, M S_{\mathrm{e}}=465,379, p<.0001\right]$ and hemisphere $\left[F(1,49)=10.169, M S_{\mathrm{e}}=97,389.5\right.$, $p<.0025$ ] but no interaction between hemisphere and condition $(F<1)$. Despite the interaction being nonsignificant, we performed the planned $t$ tests to fully explore these data. These $t$ tests performed on the difference between the RTs for the no-change and the no-rotation conditions revealed that the $\mathrm{LH}[t(49)=2.508, p<.01]$, but not the RH $[t(49)=1.251, p>.05]$, incurred a cost to recognition time from the no-change-rotation that was significantly greater than zero. In addition, Scheffé tests revealed that RTs in the change-rotation condition were significantly longer than those in the no-change-rotation and the no-rotation conditions for both hemispheres (all $p$ s $<.0005)$. Scheffé tests also revealed that despite a main effect of hemisphere in the RT data, only the norotation condition showed a significant difference between the two hemispheres (no-change-rotation, $p<.01$; no-rotation, $p>.05$; change-rotation, $p>.05$ ).

\section{Discussion}

We found that recognition was VD regardless of which hemisphere the object was presented to. The LH experienced a greater cost to recognition sensitivity than did the RH when an object was rotated in depth with no change to the visible parts. In addition, there was some mixed evidence suggesting that the RT data also reflected a similar trend; despite a nonsignificant interaction, planned $t$ tests suggested that the LH experienced a greater cost than did the $\mathrm{RH}$ in the no-change-rotation condition. These results stand in contrast to those of Burgund and Marsolek (2000), who found a stronger viewpoint effect in the RH than in the LH. One main difference between our study and that of Burgund and Marsolek
(2000) relates to object familiarity; whereas objects used in their study were familiar, in our study, novel objects were used. As was noted above, it has been suggested that the specific subsystem is specialized for processing novelty, which would predict better overall performance in the RH than in the LH on our task, due to the more effective nature of the specific subsystem in the RH (Burgund \& Marsolek, 2000). Specialization for novelty, however, should not by itself result in a differential viewpoint effect (i.e., a smaller viewpoint effect in the RH than in the LH); rather, a main effect would be predicted, because the RH should more efficiently process novel stimuli regardless of viewpoint. Thus, the DNS theory does not seem to be sufficient to account for our results.

It could be argued that the letter presented to ensure central fixation before presentation of the test object may have been responsible for the greater cost experienced by the LH in the RT data. For example, the letter may have recruited lexical resources in the $\mathrm{LH}$ to a greater degree than in the RH. However, this explanation is unlikely for two reasons: First, this task was trivially easy, providing central fixation was maintained; second, the main effect of hemisphere in these RT data appears to be carried by a single condition-the no-change-rotation. More important, if this task had compromised the ability of the $\mathrm{LH}$, we would expect that performance in the LH would suffer significantly within each condition, not just in one particular condition (no-change-rotation). The difference between performances in the other conditions across the hemispheres was nonsignificant, and the main effect of hemisphere appeared to be dependent on the norotation condition. The small nonsignificant difference in RTs in the no-rotation and the change-rotation conditions between the hemispheres might reflect a small speed-sensitivity tradeoff. Indeed, although performance in these conditions was slightly slower, it was also slightly more sensitive. As a caveat, it should be noted that this trend was too small to be detected statistically $(p>.05)$; yet it is suggestive.

However, other theoretical accounts of hemispheric differences in cognitive processing may be useful in explaining our results. Neuropsychological evidence from patients with LH lesions who are impaired at semantic categorization tasks suggests that semantic information is utilized primarily by the LH (Warrington \& Taylor, 1978). Semantic information, as available in the naming task with familiar objects used by Burgund and Marsolek (2000), may reduce viewpoint effects in the LH. However, when semantic information is not available, as in the case of novel object recognition, the LH, like the RH, may exhibit VD object recognition performance. The link between RH damage and agnosia has led many to believe that the RH plays a critical role in object recognition (Davidoff \& Warrington, 1999; Warrington \& James, 1991; Warrington \& Taylor, 1978). This leads us to suggest that visual processing in the LH may be augmented by semantic processing, which assists in achieving object constancy. Therefore, when novel stim- 
uli without semantic associations are viewed, performance is differentially impaired relative to the RH. The difficulty the LH has in utilizing the modified information in the no-change-rotation condition, as compared with the $\mathrm{RH}$, would result in a differential impairment in object recognition in this hemisphere. However, both hemispheres may be equally affected by the changerotation, since the superior abilities of the RH in achieving object constancy may be unable to compensate for the physical absence of information.

Could the LH VI results obtained by Burgund and Marsolek (2000) be due to semantic, nonvisual information? One clue that this may be so is that equivalent performance was found in same-view, different-view-sameparts, and different-view-different-parts conditions. This finding stands in contradistinction to most other object recognition studies, which have shown increased costs for object rotations that change the visible parts, as compared with rotations that keep the same parts visible (e.g., Biederman \& Gerhardstein, 1993; Hayward, 1998; Hayward \& Tarr, 1997). Although Burgund and Marsolek (2000) argued that such results might be expected from a model of object recognition in which more general features than parts are stored, no such model is currently proposed in the literature.

The DNS theory does not postulate a role for semantic information in contributing to the viewpoint invariance performance of the $\mathrm{LH}$ and, instead, states that the performance of both the LH and the RH subsystems rely only on structural (visual) information (Marsolek, 1999). The main evidence that LH VI object recognition is visual rather than semantic comes from a study by Marsolek (1999), which showed that an object picture was primed by a different object with the same name as well as it was primed by itself, but better than it was primed by its name. Thus, it was reasoned, the priming could not simply be semantic, since the name provided an appropriate semantic cue but produced less priming than did a picture of the object. However, that study did not determine whether the picture and the word resulted in similar levels of semantic activation. If the processing of pictures results in deeper semantic activation than does the processing of words (which may be a partial cause of the picture superiority effect; Shepard, 1967), priming between two pictures might be expected to be greater than priming of a picture by a word in the $\mathrm{LH}$, but not in the RH, just as Marsolek (1999) found. We do not intend to claim that this account is a definitive explanation for the greater priming for pictures than for names in Marsolek (1999) but simply that other explanations are possible and, so, the question remains an empirical one.

\section{EXPERIMENT 2}

There is a long history behind the idea that the LH dominates the linguistic or verbal functions of the human brain, with evidence from lesion studies (Broca, 1861; see
Kann, 1950), behavioral studies (Hellige, 2001), brain stimulation (Ojemann, 1983), and more recently, neuroimaging (Demb et al., 1995; Wagner, Desmond, Demb, Glover, \& Gabrieli, 1997). In particular, a clear role for the left inferior frontal gyrus (LIFG) in semantic processing has been established (see Bookheimer, 2002, and Cabeza $\&$ Nyberg, 2000, for reviews). Therefore, it would not be surprising that the semantic information typically associated with familiar objects may preferentially influence visual processing in the $\mathrm{LH}$.

Like Burgund and Marsolek (2000), we attribute the incompatibility of the results in Experiment 1 with the predictions of the DNS theory (Marsolek, 1999) to our use of novel objects. However, rather than assume that the causal variable is novelty per se, we specifically propose that the difference is that our objects lack semantic information. According to this view, if participants were to associate semantic information with novel objects, VI performance in the LH should result. In Experiment 2, we tested this prediction directly by training participants to associate semantic features with different novel objects and then asking the participants to perform a sequentialmatching task on these same depth-rotated 3-D novel objects. In addition, a control group of participants was exposed to the novel objects under similar conditions, but without the semantic information, and later performed the same sequential-matching task as the semanticstrained participants.

\section{Method}

Participants. Fifty-nine self-reported (strongly) right-handed students at Vanderbilt University participated for payment or course credit. The participants were asked a series of questions about their hand preference in everyday activities other than writing. Only those participants who reported that their right-hand was dominant both in writing and in other everyday activities were selected to participate in this experiment. Groups of 30 (15 male) and 29 (14 male) participants were placed in the semantic and the control conditions, respectively. The participants gave informed consent according to procedures approved by the Vanderbilt University Ethics Committee.

Materials. Four highly similar novel objects (YUFOs; Figure 3), created using FormZ (Autodessys Inc., Columbus, OH) and rendered with a blue tint in Lightscape (Lightscape Technologies, San Jose, CA) to create natural looking illumination conditions, were selected for this experiment. The four objects were rendered at an arbitrary canonical orientation $\left(0^{\circ}\right)$, as well as from four other viewpoints generated by progressive $30^{\circ}$ rotations in depth: $30^{\circ}, 60^{\circ}, 90^{\circ}$ (approximating the mirror image of $0^{\circ}$ ), and $120^{\circ}$ around the vertical axis. A pool of 16 features, also used in previous experiments (Gauthier, James, Curby, \& Tarr, 2003), was used ("fast," "flexible," "friendly," "cold," "rare," "sweet," "fragile," "hollow," "nervous," "sticky," "soft," "wet," "loud," "heavy," "nocturnal," and "strong”).

Design and Procedure. The experimental condition began with a study phase in which the participants associated three features with each of four YUFOs (see Gauthier et al., 2003). The sets of semantic associations were of two types: for some participants, each feature was associated with only one YUFO (e.g., "small, flexible, and wide"; "sticky, fragile, and hollow"; "friendly, rare, and strong"; etc.). For other participants, each feature could be associated with several different YUFOs, and the YUFOs differed only in the particular conjunction of features associated with them (e.g., 

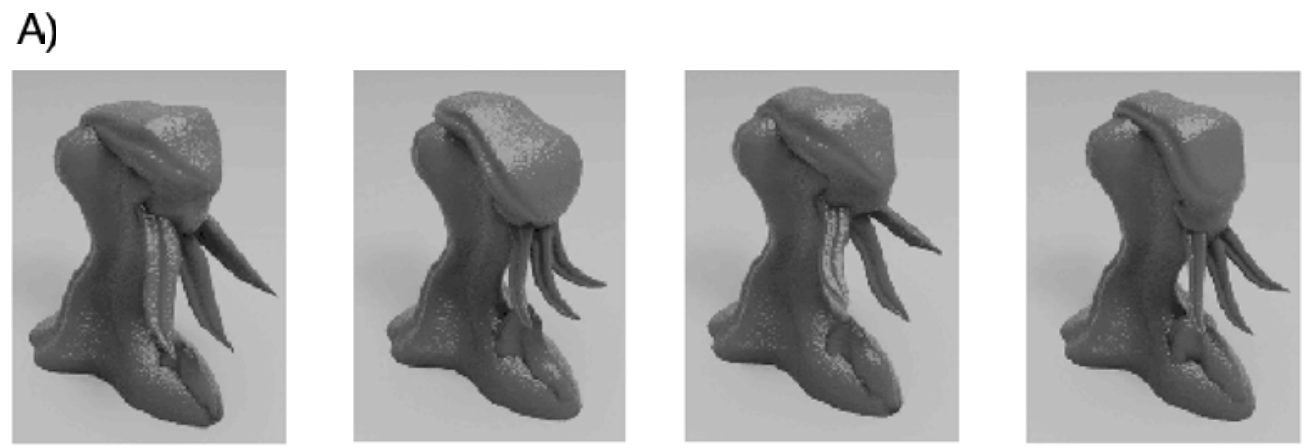

B)
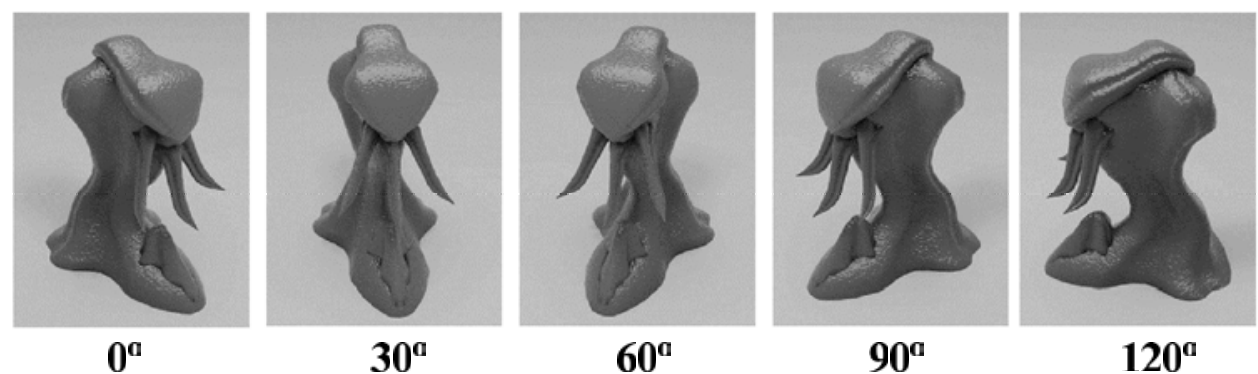

Figure 3. (A) The four novel objects used as stimuli in Experiment 2. (B) Five different views $\left(0^{\circ}, 30^{\circ}, 60^{\circ}\right.$, $90^{\circ}$, and $120^{\circ}$ ) of the objects were used for the viewpoint manipulation.

"small, flexible, and wide"; "small, wide, and cold"; "flexible, wide, and cold"; etc.). For each type, 19 orthogonal combinations of features were used.

The participants in the experimental (semantic) group were shown each object two times simultaneously with two features from its three-feature concept for $5 \mathrm{sec}$. The participants then answered 16 questions about the association between pairs of features and the objects (e.g., "Is this one COLD and FLEXIBLE?"). Following this, the two objects where shown three times simultaneously with all three features from their associated concept for $5 \mathrm{sec}$. The participants were then asked to answer 24 questions about the association between single features and objects ("Is this one STICKY?"). After this, the remaining two objects were shown three times simultaneously with their three features from the associated concept for $5 \mathrm{sec}$. During the next stage, the participants answered 50 questions about the associations between single features and all four objects. This first stage of the study phase (110 trials) was repeated, for a total of 220 study trials. To facilitate learning, at four points during this procedure, the participants wrote down all the features they could remember. Finally, the participants performed matching trials in which three features were displayed and three of the four objects were presented at the bottom of the screen with associated numeric responses (1-3). After a minimum of 24 matching trials, the participants cycled through blocks of (12) trials until they made fewer than two mistakes in one block.

During the study phase, the participants in the control group viewed the objects in the same context as those in the semantic group, except that instead of answering questions about semantic associations, they made a one-back similarity judgment for the same number of trials. The participants were asked to respond using a keypress, as to whether the object was very similar, similar, or dissimilar to the object that had appeared before it. In the final part of the study phase, in which the participants in the semantic group performed a semantic matching-to-sample judgment, the participants in the control group made a visual matching-to-sample judgment. The control participants in this part were presented with a single object $(250 \mathrm{msec})$, followed by a mask $(500 \mathrm{msec})$, and then three objects were presented on the screen and remained there until the participant pressed a key as to which of the three objects was the same as the one shown prior to the mask. Like the semantic group, after a minimum of 24 matching trials, the participants cycled through blocks of (12) trials until they made fewer than two mistakes in one block.

Following the study phase, in which the YUFOs were shown only in their canonical viewpoint, the participants in both the semantic and the control groups performed the same sequential-matching task on depth-rotated YUFOs. On each trial, a fixation cross appeared for 1,000 msec, followed by a YUFO for 1,500 msec and a second YUFO for $180 \mathrm{msec}$, which appeared either to the right or to the left of the central fixation cross and was masked (200 msec). The participants were asked to respond (same or different) as quickly as they could, using the keypad. The first YUFO was always shown at $0^{\circ}$, whereas the second YUFO could be at any of the five viewpoints $\left(0^{\circ}, 30^{\circ}, 60^{\circ}, 90^{\circ}\right.$, or $\left.120^{\circ}\right)$. Half the trials showed different YUFOs. For both same and different trials, the second object appeared equally often on the left and right sides of the display. The participants performed six blocks of 40 trials, for a total of 240 trials.

\section{Results}

Data from 5 participants were excluded from the analysis due to near-chance performance. Data from an additional participant were excluded due to a failure to keep his head in the chinrest. In addition, all the trials with an RT below $200 \mathrm{msec}$ were excluded ( $<5 \%$ of the trials). An ANOVA with hemisphere ( $\mathrm{LH}$ or $\mathrm{RH})$ and viewpoint $\left(0^{\circ}, 30^{\circ}, 60^{\circ}\right.$, or $\left.120^{\circ}\right)$ as the within-subjects factors and 

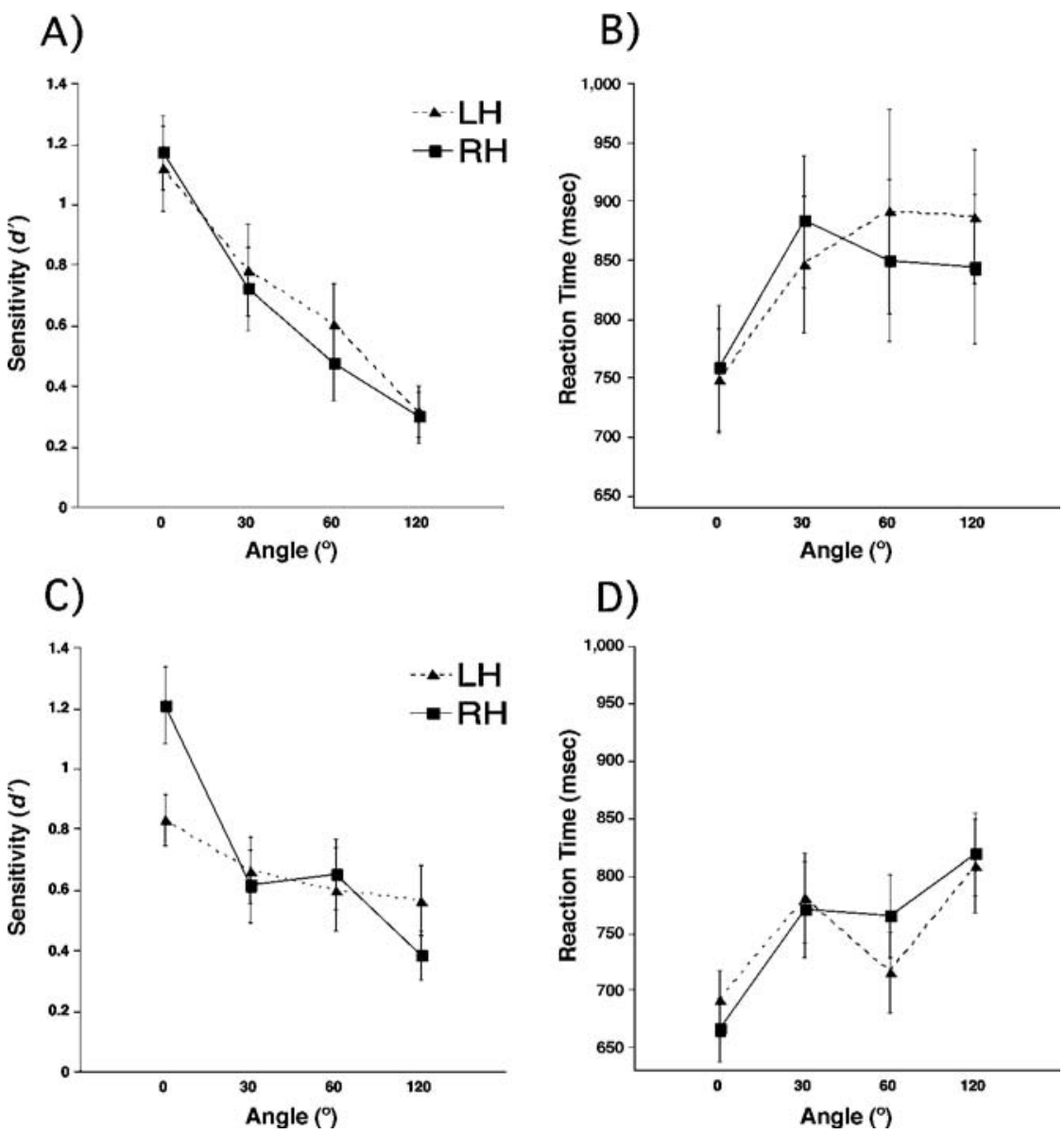

Figure 4. Mean sensitivity and reaction time in the lateralized perceptual-matching task (A and B) for participants who did not learn the semantic associations for the novel objects (control condition) and ( $C$ and $D$ ) for those who learned semantic associations for the novel objects (semantic condition). The $90^{\circ}$ view (not shown) approximated the mirror view of the studied view, leading to anomalous performance $\left(\mathrm{LH}, d^{\prime}=1.13, S E=0.12, \mathrm{RT}=762.0 \mathrm{msec}\right.$, $\left.S E=41.0 ; \mathrm{RH}, d^{\prime}=0.80, S E=0.10, \mathrm{RT}=736.3 \mathrm{msec}, S E=33.5\right)$, relative to the surrounding views.

semantic overlap as a between-subjects factor was performed on the sensitivity $\left(d^{\prime}\right)$ and RT data from the participants in the semantic group. This analysis failed to show a significant effect of semantic condition (i.e., semantically overlapping vs. semantically different associations) or any interaction between this factor and any other (all $p \mathrm{~s}>.28$ ). These data were therefore collapsed over the two conditions. The resulting mean sensitivity $\left(d^{\prime}\right)$ and RTs for the RH and the LH are shown in Figure 4 . The overall sensitivity level for the participants in the semantic (0.745) and the control (0.718) groups was very similar, suggesting that the differences in the study phase procedure did not provide one group with an overall advantage in the matching task.

The $90^{\circ}$ viewpoint - approximating the mirror image of the studied view-was anomalous, resulting in better performance, relative to the surrounding viewpoints, in both hemispheres. In particular, the LH experienced a large peak in performance at this viewpoint for the semantic group, with performance exceeding that for the trained view. This increase in performance, although surprisingly large for the LH semantic group, was expected, given that perceptual processes are known to be more robust to mirror image matching than to changes in viewpoint (Biederman \& Cooper, 1991a; Corballis, Zbrodoff, Shetzer, \& Butler, 1984; Tarr \& Pinker, 1989). Since the issue of the robustness of mirror image recognition is not central to the question motivating this study, and because of the potential for this unique view to disguise the linear effect of viewpoint in statistical analyses, the $90^{\circ}$ viewpoint was removed from further analyses. ${ }^{2}$

Control condition. The participants in the control condition responded more quickly and with greater sensitivity at smaller deviations from the studied view $\left(0^{\circ}\right)$. However, the participants in this condition incurred approximately the same cost to recognition in both hemi- 
spheres, due to the change in viewpoint, and this effect was of similar magnitude for both the male and the female participants.

The above conclusions are supported by mixed ANOVAs, with hemisphere (LH or RH) and viewpoint $\left(0^{\circ}, 30^{\circ}, 60^{\circ}\right.$, or $\left.120^{\circ}\right)$ as the within-subjects factors and sex as a between-subjects factor, performed on these sensitivity $\left(d^{\prime}\right)$ and RT data. The analysis of the RT data showed no main effect of $\operatorname{sex}[F(1,23)=1.547$, n.s. $]$ and no interactions between sex and hemisphere $[F(1,23)=$ 1.587 , n.s.], sex and viewpoint $(F<1)$, and sex, viewpoint, and hemisphere $(F<1)$. The same pattern was found for sensitivity, except that the interaction between sex and hemisphere was significant $[F(1,23)=5.487$, $p<.05]$; again there was no effect of $\operatorname{sex}[F(1,23)=$ 2.218 , n.s.] and no interaction between sex and viewpoint $(F<1)$, and the three-way interaction of sex, viewpoint, and hemisphere was not significant $(F<1)$. The interaction between sex and hemisphere was driven by lower performance in the $\mathrm{LH}$ for the females and was not a differential effect of viewpoint, and thus, the following analyses were performed on data collapsed over sex. There was a strong linear effect of viewpoint for both $d^{\prime}$ $\left[F(1,23)=41.767, M S_{\mathrm{e}}=17.916, p \leq .0001\right]$ and $\mathrm{RT}$ $\left[F(1,23)=14.296, M S_{\mathrm{e}}=279,156, p<.005\right]$, but no main effect of hemisphere $\left(d^{\prime}, F<1\right.$; RT, $\left.F<1\right)$ and no interaction between hemisphere and viewpoint $\left(d^{\prime}, F<\right.$ $1 ; \mathrm{RT}, F(1,23)=1.308$, n.s. $)$.

Semantic condition. The participants in the semantic group, like those in the control group, responded more quickly and with greater sensitivity to smaller deviations from the studied view $\left(0^{\circ}\right)$. However, unlike in the control condition, the RH incurred a greater cost to recognition than did the $\mathrm{LH}$ following the change in viewpoint.

An ANOVA with hemisphere (LH or RH) and viewpoint $\left(0^{\circ}, 30^{\circ}, 60^{\circ}\right.$, or $\left.120^{\circ}\right)$ as the within-subjects factors and sex as a between-subjects factor was performed on these sensitivity $\left(d^{\prime}\right)$ and RT data. This analysis showed no main effect of sex and no interaction between sex and viewpoint, sex and hemisphere, or sex, hemisphere, and viewpoint for both sensitivity (sex, $F<1$; sex $\times$ hemisphere, $F=2.24, M S_{\mathrm{e}}=0.224$, n.s; sex $\times$ viewpoint, $F<1$; sex $\times$ viewpoint $\times$ hemisphere, $F<1$ ) and RT (sex, $F=1.805, M S_{\mathrm{e}}=352,091$, n.s.; sex $\times$ hemisphere, $F=1.03, M S_{\mathrm{e}}=14,751.3$, n.s; sex $\times$ viewpoint, $F<1$; sex $\times$ viewpoint $\times$ hemisphere, $F<1$ ). Therefore, the following analyses were performed on sensitivity and RT collapsed across sex.

Like the control group, the ANOVA performed on the data from the semantic group revealed a strong linear effect of viewpoint both for sensitivity $[F(1,28)=320.65$, $\left.M S_{\mathrm{e}}=3.933, p \leq .0001\right]$ and for RT $[F(1,28)=26.812$, $\left.M S_{\mathrm{e}}=394,066, p \leq .0001\right]$, but no main effect of hemisphere $\left(d^{\prime}, F<1\right.$; RT, $\left.F<1\right)$. However, unlike for the control group, there was a significant interaction between hemisphere and viewpoint for sensitivity $[F(1,28)=$ $\left.3.946, M S_{\mathrm{e}}=1.22, p<.01\right]$, but not for RT $[F(1,28)=$ 1.09, n.s.]. An ANOVA performed on the sensitivity data from the LH and the RH conditions separately showed that the linear effect of viewpoint approached significance in the LH $\left[F(1,28)=3.428, M S_{\mathrm{e}}=1.068, p=\right.$ $.0747]$. However this effect only reached significance in the $\mathrm{RH}\left[F(1,28)=34.258, M S_{\mathrm{e}}=8.604, p \leq .0001\right]$.

\section{Discussion}

Consistent with the DNS theory, the sensitivity measure in Experiment 2 demonstrated the predicted pattern of viewpoint dependence in the $\mathrm{RH}$ and relatively VI performance in the LH, but only in those participants who had learned semantic associations for the objects; those in the control condition showed VD performance in both hemispheres. The participants in both conditions not only received equivalent exposure, but also performed equally well on the matching task. This suggests that the reduced viewpoint dependence exhibited by the LH for objects that have acquired semantics is due to a qualitative difference in the nature of the processing, rather than to a difference in task difficulty.

The interaction between viewpoint and hemisphere in the semantic group in Experiment 2 was evident only in the sensitivity analysis, and not in the RT analysis. The failure of the RT data to reflect this effect may be in part due to the generally larger variability in the RT measure (see Figure 4). The low sensitivity exhibited by both the semantic and the control groups clearly suggests that the participants found the matching task quite difficult and made numerous errors, which resulted in many trials being excluded from the RT analysis. It is likely that the fewer number of trials contributed to the increased variability and, thus, reduced the reliability of this measure of performance. In addition, the difficulty of the task may have also encouraged the participants to apply different strategies, possibly contributing to the variability and the resulting instability in the RT measure.

Although the control group in Experiment 2 replicated the finding of VD performance in both hemispheres for objects without learned semantics, we did not find the greater viewpoint effect in the LH that we found in Experiment 1. However, the interaction in Experiment 1 was driven by the larger cost to the LH in the no-changerotation and did not extend to the change-rotation condition, where both hemispheres experienced a similar cost due to the rotation. In Experiment 2, the number of parts visible at each $30^{\circ}$ rotation was not controlled, but many of the rotations resulted in a change in which parts were visible. Unfortunately, Experiment 2 cannot address why there is a greater cost to recognition in the $\mathrm{LH}$ for the no-change-rotation condition, because part visibility and rotation condition were confounded; larger rotations generally resulted in a greater change to the parts visible. We did not include this manipulation in Experiment 2 , because the finding of a greater cost due to the no-change rotation in the $\mathrm{LH}$ was tangential to our central hypotheses. It may be interesting to follow up on this finding in future studies in which the visibility of parts is controlled, as in Experiment 1. 
The unique nature of the $90^{\circ}$ view, due to its approximating the mirror image of the $0^{\circ}$ view, rendered it ill suited to analyses exploring the linear effect of viewpoint. However, a closer examination of performance at this view in isolation may provide some insight into the nature of object processing across the hemispheres. The advantage of the $90^{\circ}$ view, relative to neighboring views, was observed in both hemispheres; however, it was clearly stronger in the LH. The LH's large peak in performance at this viewpoint for the semantic group may be linked to the processing style of this hemisphere. On the basis of the results from a study of a callosotomy patient, it has been suggested that the fundamental difference between the LH and the RH with regard to basic visual processing is that the LH is directed toward pattern recognition at the expense of spatial information (Funnell, Corballis, \& Gazzaniga, 1999). This lack of encoding of spatial information by the LH may provide an advantage for object mapping when the view approximates the mirror reverse. The changes incurred by a rotation resulting in a transformation of only the spatial information (i.e., a rotation where the same parts are still present) may go undetected in the spatially ignorant LH. This explanation provides an alternative explanation for VI performance in the LH (Burgund \& Marsolek, 2000); however, it cannot account for the lack of VI performance in the absence of semantic associations found in Experiment 2.

Another interesting result that may provide insights into the nature of object recognition is the semantic group's lower performance in the $\mathrm{LH}$ at the $0^{\circ}$ viewpoint. This difference may occur because the nature of the task at $0^{\circ}$ leads to a disadvantage for the LH. It is possible that the semantic input may actually interfere with the picture matching that is sufficient in the $0^{\circ}$ view condition; that is, the same input that helps to produce VI performance may interfere in a task that requires simply picture matching. Evidence consistent with this explanation comes from a recent study, also using YUFOs as stimuli, which found that participants who learned semantic associations (e.g., sticky, nervous, strong) for novel objects performed significantly worse on a subsequent matching task than did those who learned only a nickname (e.g., Mike; James \& Gauthier, 2004). Importantly, the objects for which the participants had learned names did not produce activity (relative to untrained objects) in the left frontal region associated with semantic retrieval, whereas the objects for which semantics had been learned did. This explanation is obviously very tentative and raises interesting questions that we intend to explore in future studies.

\section{GENERAL DISCUSSION}

Within the context of the current literature, the results of these experiments suggest that VI processing in the LH may be due, at least partially, to a specialization for semantic processing. The LH-in particular, the LIFGhas been implicated in semantic processing (see Book- heimer, 2002, and Cabeza \& Nyberg, 2000, for reviews). An area of specific interest in this region, likely corresponding to Brodmann's Area 47, appears to be involved in retrieving semantic information (Demb et al., 1995). This area is believed to be responsible for a general semantic retrieval process, since it is active under a variety of conditions, including both classification and generation tasks (Cabeza \& Nyberg, 2000).

This striking left-lateralization of semantic processing is observed not only for verbal stimuli, but also with pictorial stimuli. Vandenberghe, Price, Wise, Josephs, and Frackowiak (1996) investigated semantic processing, using PET, and found greater activity in the LIFG for both picture and word semantic tasks (associative semantic judgments and visual semantic judgments), as compared with a perceptual baseline (perceptual size judgments). In addition, an fMRI repetition-priming study has shown evidence that this anterior LIFG area not only is involved in semantic retrieval, but also is modality independent (Wagner et al., 1997). In this study, LIFG activity was measured during repeated, relative to initial, semantic processing of words (word-to-word semantic repetition priming) and of pictures (picture-topicture repetition priming).

A recent fMRI study (Vuilleumier, Henson, Driver, \& Dolan, 2002) has demonstrated that activity in the fusiform and LIFG regions showed repetition priming for real objects, but not for nonsense (novel) objects. The lack of priming for the novel objects (which lack semantic associations) in the LIFG is consistent with its role in semantic retrieval. In addition, this study also showed repetitionpriming-induced decreases in activity across different viewpoints for real objects in the left fusiform gyrus, but not in the right fusiform gyrus. These results are consistent with the DNS theory, although in this case, as in previous experiments, semantic information may have facilitated the LH's VI performance. Conducting this experiment with novel objects would provide the necessary test for the DNS theory, to determine whether the VI performance of the LH is facilitated by the retrieval of semantic information in the LIFG, as our study suggests.

An important consideration is whether the results from Experiment 2 can clearly distinguish between our hypothesis that there is a role of semantic information in VI performance and the DNS theory. The DNS theory allows for the possibility that interactive feedback from conceptual subsystems may help the abstract subsystem learn to categorize different input shapes together and activate the same output representation (Marsolek, 1999). Thus, the crucial distinction between the two hypotheses is whether the VI performance of the LH relies on semantic input during recognition processes or, alternatively, is a product of structural representations that follow feedback from semantic representations during the study phase.

With neuroimaging studies, an attempt can be made to provide some insight into this issue. For example, a recent fMRI study in which a perceptual-matching task was used with the same novel objects as those in Exper- 
iment 2 showed increased activity in the anterior LIFG for objects with learned semantic associations, as compared with those with no learned associations (James \& Gauthier, 2004). Importantly, areas that we suggest may be providing VI input during recognition were active during a one-back perceptual-matching task in which the retrieval of the semantic information associated with the objects was not necessary. Therefore, the difference in activation in the LIFG during this task suggests that this semantic-processing area may be activated automatically. This result provides a possible LH locus for the findings of our study and suggests that the LH may provide input during recognition. One must be cautious when interpreting these results, since the increased activation in frontal semantic areas could be due to feed-forward mechanisms from visual areas after recognition has taken place. However, this is unlikely, given our results and those of others (Gauthier et al., 2003), demonstrating that semantics can influence basic perceptual performance and, therefore, suggesting that the influence is likely to occur before the recognition process is complete.

Techniques such as transmagnetic stimulation could potentially differentiate between the two alternative hypotheses addressed in this article, since they would predict different results if a (right-handed) participant were to have his or her left inferior frontal cortex stimulated in such a way as to induce a temporary lesion while a matching task was performed. The DNS theory would predict that there would be no change in performance; however, we would predict that the $\mathrm{LH}$ would exhibit more VD performance when the task was performed, after receiving the stimulation to the left inferior frontal region.

Although this study, in isolation, cannot easily distinguish between the two alternative hypotheses, when it is considered in combination with recent imaging studies that have found results consistent with automatic semantic mediation during visual recognition, a strong case can be made for the influence of semantics during object recognition. This conclusion need not be viewed as antithetical to the DNS theory. Burgund and Marsolek (2000) suggested that the specific subsystem is advantaged when novel objects are viewed, due to its superior ability to encode holistic information, which they suggested is typically where novel information lies. Equally, however, an abstract subsystem that interfaced with an LH-localized semantics system could give rise to the VI performance found in Burgund and Marsolek's (2000) experiment, as well as in Experiment 2 in this article. Clearly, further research is needed to tease apart these theoretical alternatives; indeed, both may well be true.

\section{CONCLUSION}

The experiments outlined above demonstrate not only that there is an LH region responsible for semantic processing, but also that this region is activated in a perceptual task with novel objects, providing observers have learned semantic information for these objects. There- fore, interactions between semantic and visual processing may be responsible for a reduced cost of viewpoint changes for familiar objects in the LH. Our results suggest that visual processing in the left and that in the right cerebral hemispheres are mediated by qualitatively similar systems. We found that when novel objects are used in a visual-matching task across viewpoints, both hemispheres show VD recognition. However, associating semantic information with novel objects reduced the viewpoint effect only in the LH. Thus, we propose that the LH VI performance found by Burgund and Marsolek (2000) may be the result of an interaction with LH systems (such as the LIFG) processing semantic information. This result highlights the importance of using novel objects to investigate perceptual phenomena and will, hopefully, lead to more research on the similarities and differences in the recognition of novel and familiar objects, which in turn should lead to more comprehensive theories of object recognition.

\section{REFERENCES}

BIE DERMAN, I. (1987). Recognition-by-components: A theory of human image understanding. Psychological Review, 94, 115-147.

BIEDERMAN, I., \& BAR, M. (1999). One-shot viewpoint invariance in matching novel objects. Vision Research, 39, 2885-2899.

BIEDERMAn, I., \& COOPER, E. E. (1991a). Evidence for complete translational and reflectional invariance in visual object priming. Perception, 20, 585-593

BIEDERMAN, I., \& COOPER, E. E. (1991b). Object recognition and laterality: Null effects. Neuropsychologia, 29, 695-702.

Biederman, I., \& CoOper, E. E. (1992). Size invariance in visual object priming. Journal of Experimental Psychology: Human Perception \& Performance, 18, 121-133.

BIEDERMAN, I., \& GERHARDSTEIN,P. C. (1993). Recognizing depth-rotated objects: Evidence and conditions for three-dimensional viewpoint invariance. Journal of Experimental Psychology: Human Perception \& Performance, 19, 1162-1182.

BOOKHE IMER, S. (2002). Functional MRI of language: New approaches to understanding the cortical organization of semantic processing. Annual Review of Neuroscience, 25, 151-188.

Burgund, E. D., \& MARSOLEK, C. J. (1997). Letter-case-specific priming in the right cerebral hemisphere with a form-specific perceptual identification task. Brain \& Cognition, 35, 239-258.

Burgund, E. D., \& MARsole K, C. J. (2000). Viewpoint-invariant and viewpoint-dependent object recognition in dissociable neural subsystems. Psychonomic Bulletin \& Review, 7, 480-489.

CABEZA, R., \& NYBERG, L. (2000). Imaging cognition: II. An empirical review of 275 PET and fMRI studies. Journal of Cognitive Neuroscience, 12, 1-47.

CARPENTER, R. H. S. (1981). Oculomotor procrastination. In D. F. Fisher, R. A. Monty, \& J. W. Senders (Eds.), Eye movements: Cognition and visual perception. Hillsdale, NJ: Erlbaum.

Cooper, E. E., Biederman, I., \& Humme L, J. E. (1992). Metric invariance in object recognition: A review and further evidence. Canadian Journal of Psychology, 46, 191-214.

Corballis, M. C., Zbrodoff, N. J., Shetzer, L. I., \& Butler, P. B. (1984). Decisions about identity and orientation of rotated letters and digits. Memory \& Cognition, 6, 98-107.

DAVIDOFF, J., \& WARrington, E. K. (1999). Apperceptive agnosia: A deficit of perceptual categorisation of objects. In G. W. Humphreys (Ed.), Case studies in the neuropsychology of vision (pp. 59-79). Hove, U.K.: Psychology Press.

Demb, J. B., Desmond, J. E., Wagner, A. D., Vaidya, C. J., Glover, G. H., \& GABRIELI, J. D. E. (1995). Semantic encoding and retrieval in the left inferior prefrontal cortex: A functional MRI study of task 
difficulty and process specificity. Journal of Neuroscience, 15, 58705878.

FARAH, M. J. (1992). Is an object an object an object? Cognitive and neuropsychological investigations of domain-specificity in visual object recognition. Current Directions in Psychological Science, 1, 164169.

Funnell, M. G., Corballis, P. M., \& Gazzaniga, M. S. (1999). A deficit in perceptual matching in the left hemisphere of a callosotomy patient. Neuropsychologia, 37, 1143-1154.

Gauthier, I., James, T., Curby, K. M., \& Tarr, M. J. (2003). The influence of conceptual knowledge on visual discrimination. Cognitive Neuropsychology, 20, 507-523.

HAYWARD, W. G. (1998). Effects of outline shape in object recognition. Journal of Experimental Psychology: Human Perception \& Performance, 24, 427-440.

HAYWARD, W. G., \& TARR, M. J. (1997). Testing conditions for viewpoint invariance in object recognition. Journal of Experimental Psychology: Human Perception \& Performance, 23, 1511-1521.

Hayward, W. G., \& Williams, P. (2000). Viewpoint dependence and object discriminability. Psychological Science, 11, 7-12.

Hellige, J. B. (2001). Hemispheric asymmetry: What's right and what's left. Cambridge, MA: Harvard University Press.

Humme L, J. E., \& STANKIEWICZ, B. J. (1998). Two roles for attention in shape perception: A structural description model of visual scrutiny. Visual Cognition, 5, 49-79.

JAMES, T. W., \& GAUTHIER, I. (2004). Brain areas engaged during visual judgments by involuntary access to novel semantic information. $\mathrm{Vi}$ sion Research, 44, 429-439.

KANN, J. (1950). A translation of Broca's original article on the location of the speech center [Remarques sur le siège de la faculté du langage articulé, suivies d'une observation d'aphémie]. Journal of Speech \& Hearing Disorders, 15, 16-20. (Original work published 1861)

MARSOLEK, C. J. (1995). Abstract visual-form representations in the left cerebral hemisphere. Journal of Experimental Psychology: Human Perception \& Performance, 21, 375-386.

MARSOLE K, C. J. (1999). Dissociable neural subsystems underlie abstract and specific object recognition. Psychological Science, 10, 111-118.

Marsolek, C. J., Kosslyn, S. M., \& SQuire, L. R. (1992). Form-specific visual priming in the right hemisphere. Journal of Experimental Psychology: Learning, Memory, \& Cognition, 18, 492-508.

Marsolek, C. J., Schacter, D. L., \& Nicholas, C. D. (1996). Formspecific visual priming for new associations in the right cerebral hemisphere. Memory \& Cognition, 24, 539-556.

Marsolek, C. J., Squire, L. R., Kosslyn, S. M., \& Lulenski, M. E. (1994). Form-specific explicit and implicit memory in the right cerebral hemisphere. Neuropsychology, 8, 588-597.

OJEMANN, G. A. (1983). Brain organization for language from the perspective of electrical stimulation mapping. Behavioral \& Brain Sciences, 6, 189-230.

OLDFIELD, R. C. (1971). The assessment and analysis of handedness: The Edinburgh Inventory. Neuropsychologia, 9, 97-113.

PogGio, T., \& EdELmAn, S. (1990). A network that learns to recognize three-dimensional objects. Nature, 343, 263-266.
Riesenhuber, M., \& Poggio, T. (1999). Hierarchical models of object recognition in cortex. Nature Neuroscience, 2, 1019-1025.

SHE PARD, R. N. (1967). Recognition memory for words, sentences, and pictures. Journal of Verbal Learning \& Verbal Behavior, 6, 156-163.

SRINIVAS, K. (1995). Representation of rotated objects in explicit and implicit memory. Journal of Experimental Psychology: Learning, Memory, \& Cognition, 21, 1019-1036.

TARR, M. J. (1995). Rotating objects to recognize them: A case study on the role of viewpoint dependency in the recognition of three-dimensional objects. Psychonomic Bulletin \& Review, 2, 55-82.

TARR, M. J., \& BÜLTHOFF, H. H. (1995). Is human object recognition better described by geon structural descriptions or by multiple views? Comments on Biederman and Gerhardstein (1993). Journal of Experimental Psychology: Human Perception \& Performance, 21, 1494-1505.

TARR, M. J., \& GAUTHIER, I. (1998). Do viewpoint-dependent mechanisms generalize across members of a class? Cognition, 67, 73-110.

TARR, M. J., \& PINKER, S. (1989). Mental rotation and orientationdependence in shape recognition. Cognitive Psychology, 21, 233-282.

TARR, M. J., \& PINKER, S. (1990). When does human object recognition use a viewer-centered reference frame? Psychological Science, 1, 253-256

Tarr, M. J., Williams, P., Hayward, W. G., \& Gauthier, I. (1998). Three-dimensional object recognition is viewpoint dependent. $\mathrm{Na}$ ture Neuroscience, 1, 275-277.

VANDEnberghe, R., Price, C., Wise, R., Jose Phs, O., \& Frackowiak, R. S. J. (1996). Functional anatomy of a common semantic system for words and pictures. Nature, 383, 254-256.

Vuilleumier, P., Henson, R. N., Driver, J., \& Dolan, R. J. (2002). Multiple levels of visual object constancy revealed by event-related fMRI of repetition priming. Nature Neuroscience, 5, 491-499.

Wagner, A. D., Desmond, J. E., Demb, J. B., Glover, G. H., \& GABRIELI, J. D. E. (1997). Semantic repetition priming for verbal and pictorial knowledge: A functional MRI study of left inferior prefrontal cortex. Journal of Cognitive Neuroscience, 9, 714-726.

WARRINGTON,E. K., \& JAMES, M. (1991). A new test of object decision: 2D silhouettes featuring a minimal view. Cortex, 27, 377-383.

WARrington, E. K., \& TAYLOR, A. M. (1978). Two categorical stages of object recognition. Perception, 7, 695-705.

\section{NOTES}

1. A $2 \times 3 \times 2$ (hemisphere, rotation, and sex) ANOVA performed on these sensitivity and RT data showed no main effect or interaction involving the sex factor (all $p s>.15$ ). Therefore, all the analyses reported for Experiment 1 were performed on these data collapsed over sex.

2. An ANOVA performed on the $d^{\prime}$ and RT measures including data from the $90^{\circ}$ view failed to find a significant linear effect $\left[d^{\prime}, F(1,28)=\right.$ 1.476 , n.s.; RT, $F<1]$ or interaction $\left[d^{\prime}, F(1,28)=1.314\right.$, n.s.; RT, $F<1]$ with viewpoint.

(Manuscript received November 22, 2002; revision accepted for publication January 3, 2004.) 